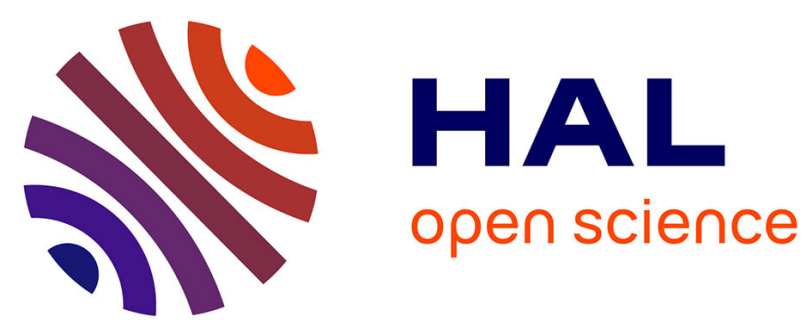

\title{
New long-range sub-structure found in the tetragonal phase of CH3NH3PbI3 single crystals
}

\author{
V.L.R. Jacques, A Gallo-Frantz, Antonio Tejeda, D Le Bolloc'h, Ferdinand \\ Lédée, Gaëlle Trippé-Allard, Damien Garrot, Pierre Fertey, Emmanuelle \\ Deleporte, Olivier Plantevin
}

\section{To cite this version:}

V.L.R. Jacques, A Gallo-Frantz, Antonio Tejeda, D Le Bolloc'h, Ferdinand Lédée, et al.. New longrange sub-structure found in the tetragonal phase of CH3NH3PbI3 single crystals. Journal of Physics D: Applied Physics, 2019, 52, pp.314001. 10.1088/1361-6463/ab202e . hal-02384710

\section{HAL Id: hal-02384710 \\ https://hal.science/hal-02384710}

Submitted on 4 Dec 2019

HAL is a multi-disciplinary open access archive for the deposit and dissemination of scientific research documents, whether they are published or not. The documents may come from teaching and research institutions in France or abroad, or from public or private research centers.
L'archive ouverte pluridisciplinaire HAL, est destinée au dépôt et à la diffusion de documents scientifiques de niveau recherche, publiés ou non, émanant des établissements d'enseignement et de recherche français ou étrangers, des laboratoires publics ou privés. 


\title{
New long-range sub-structure found in the tetragonal phase of $\mathrm{CH}_{3} \mathrm{NH}_{3} \mathrm{Pbl}_{3}$ single crystals
}

V.L.R. Jacques ${ }^{1,}{ }^{*}$, A. Gallo-Frantz ${ }^{1}$, A.Tejeda ${ }^{1}$, D. Le Bolloc' $h^{1}$, F. Lédée ${ }^{2}$, G. Trippé-Allard ${ }^{2}$, D. Garrot ${ }^{3}$, P. Fertey ${ }^{4}$, E. Deleporte ${ }^{2}$, O. Plantevin ${ }^{5}$

1- Laboratoire de Physique des Solides, CNRS-Univ. Paris-Sud, Université Paris-Saclay, Bâtiment 510, Campus d'Orsay, 91405 Orsay Cedex, France

2- Laboratoire Aimé Cotton, CNRS, Univ. Paris-Sud, ENS Paris-Saclay, Université Paris-Saclay, Bâtiment 505, Campus d'Orsay, 91405 Orsay Cedex, France

3-Groupe d'Etude de la Matière Condensée, CNRS, Université de Versailles Saint-Quentin en Yvelines, Université Paris-Saclay, 45, avenue des Etats-Unis, 78035 Versailles, France 4-Synchrotron SOLEIL, L'Orme des Merisiers, Saint-Aubin, BP48, 91192 Gif-sur-Yvette Cedex, France

5- Centre de Sciences Nucléaires et de Sciences de la Matière, CNRS-IN2P3-Univ. Paris-Sud, Université Paris-Saclay, Bâtiment 104-108, Campus d'Orsay, 91405 Orsay Cedex, France

\begin{abstract}
Hybrid organic-inorganic perovskites have become one of the most promising low-cost alternatives to traditional semiconductors in the field of photovoltaics and light emitting devices. It combines both attractive features of organic and inorganic materials within a single composite, for instance with excellent electronic properties. We used x-ray diffraction to reveal a sub-structure within $\mathrm{CH}_{3} \mathrm{NH}_{3} \mathrm{Pbl}_{3}$ single crystals. We could observe the presence of additional peaks with a square symmetry in several monocrystalline samples. We discuss these results in terms of two different models: a super-structure modulated in two in-plane orthogonal directions, and a model with tilted domains with a shallow angle of $\sim 0.6^{\circ}$. In both cases, the modulated or tilted domains appear in regions with small lattice expansion. We show that this last model appears to be the most likely to explain our observations.
\end{abstract}

\section{Introduction}

Methylammonium lead triiodide $\left(\mathrm{CH}_{3} \mathrm{NH}_{3} \mathrm{Pbl}\right.$, so-called MAPI) is a reference material at the origin of the development of perovskite-based solar cells. Its crystal structure is tetragonal at room temperature, but undergoes two structural phase transitions as a function of temperature : from orthorhombic to tetragonal at $160 \mathrm{~K}$, and from tetragonal to cubic at about $330 \mathrm{~K}[1,2]$. We address

\footnotetext{
*Corresponding author : vincent.jacques@u-psud.fr
} 
here some diffraction features that appear in the tetragonal phase at room temperature where the organic cation was shown to be orientationally disordered over four sites [3]. We believe that detailed structural analysis would help in getting some answers for puzzlingly good electronic properties obtained from crystalline soft chemistry synthesis at only $100^{\circ} \mathrm{C}$ [4]. Also, a specific property of this material is its ability to accommodate structural defects with benign impact over opto-electronic properties $[5,6]$ that should be related to some specific structural and dynamic features that are yet to be elucidated.

\section{Experiment}

In this study, we performed single-crystal x-ray diffraction to probe the crystal structure of the tetragonal phase of MAPI at room temperature. The tetragonal phase is characterized by tilted halide octahedra around the c-axis, alternatively clockwise and anticlockwise for adjacent octahedra. Compared to the high-temperature cubic phase, the cell is thus doubled along the $c$ direction and tilted by $45^{\circ}$ in the $(a, b)$ plane. The tetragonal structure of MAPI is recalled in Figia and $1 b$ in the $(b, c)$ and $(a, b)$ planes respectively. The corresponding reciprocal lattice has been calculated taking $10 \times 10 \times 10$ cells. The (okl) and (hko) reciprocal planes are shown in Fig1c and $1 \mathrm{~d}$ respectively. The reflection conditions corresponding to the $14 / \mathrm{mcm}$ group are retrieved $(h+k=2 h$ in $h k o$ and $k_{1} l=2 n$ in okl) and each peak displays a cardinal sinus squared profile due to the finite size of the cell.

Single MAPI crystals were grown using the inverse temperature crystallization method [4]. Methylammonium iodide $\left(\mathrm{CH}_{3} \mathrm{NH}_{3}\right)$ l (called MAI hereafter) was synthesized by adding dropwise $10,5 \mathrm{~mL}$ of hydriodic acid $\mathrm{HI}$ ( $57 \%$ in water, stabilized, Sigma Aldrich) to $20 \mathrm{~mL}$ of methylamine $\mathrm{CH}_{3} \mathrm{NH}_{2}$ (2 M solution in ethanol, Sigma Aldrich) at $0^{\circ} \mathrm{C}$ for 2 hours. The solvent was then evaporated at $60^{\circ} \mathrm{C}$ under vacuum using a rotary evaporator. The powder was subsequently washed several times with diethyl ether and dried overnight at $60^{\circ} \mathrm{C}$. In order to increase purity, the yellowish MAI powder was finally recrystallized in a mix of ethanol and diethyl ether. $636 \mathrm{mg}$ of MAI and $1844 \mathrm{mg}$ of lead lodide $\mathrm{Pbl}_{2}$ were dissolved in $4 \mathrm{~mL}$ of DMF (1:1 molar ratio) in a small Teflon capped vial. The vial was then placed in an oil bath at $105^{\circ} \mathrm{C}$. Single crystals of $\mathrm{MAPbl}_{3}$ start to appear at the bottom of the vial after a couple of hours. There were recovered, dried and washed with diethyl ether several times. The samples are well-faceted crystals, $\sim \mathrm{mm}^{3}$ in volume, with a (100) orientation of the as-grown surface [7]. The samples were kept under $\mathrm{N}_{2}$ gas and measured under vacuum. $A$ first experiment was performed using a Rigaku RU-30oB Cu rotating anode coupled with focusing multilayers optics, producing a $\sim 800 \mu \mathrm{m}$ beam size on the sample at $8.04 \mathrm{keV}$. The sample was mounted on a Huber eulerian 4-circle diffractometer and detection was performed with a Nal 
scintillator in the vertical scattering plane. Slits were inserted just before the detector, $\sim 70 \mathrm{~cm}$ downstream of the sample, to get better angular resolution. The specular 400 reflection was measured along the longitudinal direction performing a $\theta-2 \theta$ scan (see Fig 2a). An additional feature can be observed on the lower-angle side of the 400 Bragg reflection, approximately 20 times weaker than the Bragg reflection. For reproducibility we performed the same kind of measurements with 3 different single crystals.

To get a better understanding of this additional signal measured along the $\theta-2 \theta$ scans, we recorded a $3 \mathrm{D}$ reciprocal volume around the 400 reflection. To do so, we recorded the diffracted intensity along the three non-colinear directions of reciprocal space, making $\omega / \chi$ maps at several $2 \theta$ positions of the detector. A range of 3 degrees was explored in the two directions of the angular $\omega / \chi$ maps, with steps of $37.5 \mathrm{mdeg}$, and a range of 1 degree was explored in the $2 \theta$ directions, with steps of 0.1 deg. The use of slits before the detector insured to have a good resolution in all directions of reciprocal space. The intensities were thus recorded in a $3 \mathrm{D}$ angular volume that was then converted

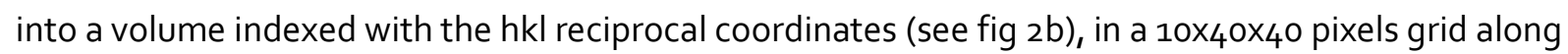
$\mathrm{h}, \mathrm{k}$ and I respectively. Note that the [010] direction of the crystal was perpendicular to the vertical scattering plane.

The measured intensity, plotted in the $3 \mathrm{D}$ hkl volume, is displayed in Figza. Several intensiy isosurfaces are displayed up to 3000 cts (see Fig 3a). The crystal truncation rod (CTR) is visible along the $\mathrm{h}$ direction of reciprocal space, as expected, as well as the 400 Bragg reflection, which lies inside the upper yellow region. However, the additional feature that was visible on the $\theta-2 \theta$ scan of Figra manifests as additional peaks that are well separated one from the other in the plane $h=3.98$ of reciprocal space. To get a better view of how those peaks are arranged, three planes at constant $\mathrm{h}$ values are displayed: the one in which the 400 Bragg reflection lies $(h=4)$, the one in which the superstructure peaks lie $(\mathrm{h}=3.98)$ and the one which is symmetric with respect to the 400 Bragg reflection ( $h=4.02$ ) (see Fig $3 b$ and Fig3c-e). For clarity reasons, the intensity scale is the same for all maps, with a maximum intensity fixed at $3000 \mathrm{cts}$. That is why in the plane $\mathrm{h}=4$, the 400 reflection appears wide although its maximum intensity is $\sim 2 \mathrm{e} 5 \mathrm{cts}$. In the plane $\mathrm{h}=3.98,4$ superstructure peaks appear at $\pm(\Delta \mathrm{k} \pm \Delta \mathrm{l})$, with $\Delta \mathrm{k} \approx \Delta \mathrm{l}=0.030 \pm 0.002$. This reciprocal space distance corresponds to $20 n m$ in real space. Finally in the symmetric $h=4.02$ plane, the CTR contributes to some intensity at $k=l=0$, but there is no such superstructure peak as in the $h=3.98$ symmetric plane. Note that this characteristic feature has been observed in the 3 samples that we measured. 
To complement these laboratory measurements, additional ones were performed at the undulator beamline CRISTAL of SOLEIL Synchrotron. The beam energy was set to $18.48 \mathrm{keV}$ with a $\mathrm{Si}(111)$ monochromator, and prefocused with mirrors. We then used $50 \times 50 \mu \mathrm{m}^{2}$ slits to define the beamsize on the sample. The rocking curves of the 400,800 and 402 reflections were recorded using a 2D Maxipix pixel detector ( $55 \mu \mathrm{m}$ pixel size), positioned $65 \mathrm{~cm}$ downstream of the sample, giving a resolution in q-space $\Delta \mathrm{q}=7.9210^{-4} \AA^{-1}$.

The 400 and 800 Bragg reflections were measured in the vertical diffraction plane in specular geometry as shown in Fig.2b, and the 402 asymmetric reflection was recorded out of the vertical plane, using the vertical rotation axis of the detector arm. Similar reciprocal space volumes as drawn in Fig. $2 b$ were thus recorded around the three Bragg reflections by performing $\omega$-scans. Projections of these $3 \mathrm{D}$ volumes are presented in Fig. 4. The four additional peaks appear close to all three reflections, although their relative positions vary significantly. In addition, they always appear in a plane perpendicular to the Bragg wavevector in reciprocal space and again, not in the main Bragg plane, but at lower values, similarly to the intensity maps shown in Figure 3 . It is convenient to compare the signals found on the 400 and 800 reflections, as the relationship between the two is straightforward (the 800 reflection is twice as far as the 400 reflection along the same reciprocal space line). One can see that the additional peaks are twice as far from the 800 reflection as they are on the 400 reflection. Similarly, they are twice as far one from each other than on the 400 reflection, in both transverse directions (called $\delta q_{\perp}$ and $\delta \omega$ on Fig 4 ).

This observation is very interesting and unexpected if only the usual crystallographic structure of MAPI is taken into account. Indeed, as shown in Fig1c-d, Bragg reflections are only expected at integer values of $h, k$ and I provided the conditions of existence are satisfied. The fact that additional well-localized peaks appear at other reciprocal space positions accounts for a new long-range extraorder. Another interesting feature is that they appear along $\mathrm{k}$ and I which are non-equivalent crystallographic directions in the tetragonal phase.

This observation cannot be explained by the presence of tetragonal domains in the sample. Indeed, for the 400 reflection, this would result in two different reflections along $h$, associated to the two non-equal lattice parameters of the tetragonal cell. Similarly, twin domains that were already observed in films would not produce such features, but reflections appearing at equivalent $2 \theta$ values, as no change of lattice parameter would take place.

We are here in the presence of well-defined reflections with a clear square symmetry in the plane $h=3.98$. Here, the superstructure peaks appear in a lower-h plane compared to the Bragg reflection, 
and their symmetric counterparts along $h$ with respect to the 400 reflection (at $h=4.02$ ) do not exist. This suggests that the average lattice parameter is expanded along a in the region of the sample where the extra-modulation lies. It could in particular be the case in the region located near the surface of the crystal, where surface reconstruction effects might play an important role, as was shown in $\mathrm{CH}_{3} \mathrm{NH}_{3} \mathrm{PbBr}_{3}$ single crystals [8]. Surface restructuring was also observed on faces of $\mathrm{CH}_{3} \mathrm{NH}_{3} \mathrm{PbBr}_{3}$ [9] and $\mathrm{CH}_{3} \mathrm{NH}_{3} \mathrm{Pbl}_{3}$ single crystals using STM [10]. The bromide single crystals trap density is for instance estimated about two orders of magnitude larger at the surface than in the bulk, giving rise to a strong lattice deformation [8].This deformation should however have relatively little impact on the electronic properties as the spectral shift between surface and bulk emission should not be larger than about $10 \mathrm{meV}$, as was shown using comparatively both cathodoluminescence and photoluminescence [11]. In this supposedly expanded lattice region, the presence of the superstructure reflections could find different origins.

A first explanation is to consider an array of lattice defects. Indeed, as the growth rate of MAPI single crystals is very high, dislocations are expected to be present in the sample with high density. In this scenario, the superstructure peaks would appear if those defects get periodically ordered every $\sim 20 n m$. Such an array of dislocations for instance would produce peak splitting due to out-ofphase crystallographic planes between the regions separated by dislocations. However, detecting such topological defects with a classical beam seems unlikely, because the probe has to be coherent over the typical size of the defected region to make the peak splitting visible, as it originates from an interference phenomenon [12-14]. In our case, with a classical beam generated by a laboratory rotating anode, this periodicity should not be visible. However, further structural studies should be considered before ruling out this scenario.

Another possible scenario involves a modulated phase. If the extra peaks can be described relatively to the 400 Bragg reflection, their position correspond to large wavelengths, around 30 times larger than the lattice parameters i.e. 20nm. Modulated phases are generally favorable in lowdimensional systems, displaying highly anisotropic properties in different directions of the crystal. This is for instance the case in Peierls-like charge-density-wave (CDW) systems [15] but they are also found in many other condensed matter systems like in magnetism, with helimagnetic structures, or composite materials with mixed periodicities [16]. In addition, modulated phases can be commensurate or incommensurate with the underlying lattice, which can lead to very different physical properties. For instance, incommensurate CDW systems can display non-linear transport properties in contrast to commensurate ones which are pinned due to a strong coupling to the underlying lattice. Even higher order modulations can appear in such states, with highly long-range 
soliton lattices $[17,18]$. In the present case of additional satellite reflections appearing in the $h=3.98$ plane, the modulated phase seems the most relevant to us. First, such a phase has been recently reported in the parent $\mathrm{Br}$ compound $\mathrm{CH}_{3} \mathrm{NH}_{3} \mathrm{PbBr}_{3}$ [19], with very similar additional reflections appearing in the diffractograms. However, the reciprocal space positions of the additional reflections with respect to Bragg reflections measured at room temperature here are ten times smaller than reported in $\mathrm{CH}_{3} \mathrm{NH}_{3} \mathrm{PbBr}_{3}$ (in a very limited temperature range) by Guo et al. [19], meaning that the associated periods are very different in the two cases.

For a better understanding of our measurements, we performed simulations using a 2D modulated lattice and calculated the associated diffraction maps. As shown in [20], a buckling instability could take place, with a displacement of the halide octahedra center of masses, inducing periodic modulations. Here, we took into account two modulations appearing in a simplified mono-atomic model lattice in the $(\vec{b}, \vec{c})$ plane, along $(\vec{b}+\vec{c})$ and $(\vec{b}-\vec{c})$ crystallographic directions, to account for the appearance of the measured satellite reflections. To do so, we first built a square lattice of those positions in a $(x, y)$ space coordinate, with orthonormal unitary vectors $(\vec{\imath}, \vec{\jmath})$, and allowed small displacements around the periodic positions. We model the total electron density of the system $\varrho_{t o t}(x, y)$ as the convolution between an on-site electron density and a 2D Dirac comb:

$$
\varrho_{t o t}(x, y)=\varrho_{0}(x, y) \otimes \sum_{n=1}^{N} \sum_{m=1}^{N} \delta\left(x-n a+u_{x}(x, y)\right) \delta\left(y-m a+u_{y}(x, y)\right)
$$

where $\varrho_{0}(x, y)=e^{-\left(x^{2}+y^{2}\right) /\left(2 \sigma^{2}\right)}$ is the on-site electron density, taken Gaussian in first approximation, $a$ is the lattice parameter, $N$ is the number of atomic cells, and $u_{x}(x, y)$ and $u_{y}(x, y)$ are space-dependent atomic displacements along $x$ and $y$ respectively.

We describe the two modulations with respective wave vectors $\overrightarrow{k_{1}} \|(\vec{\imath}+\vec{\jmath})$ and $\overrightarrow{k_{2}} \|(\vec{\imath}-\vec{\jmath})$, and assume that the corresponding displacements $\overrightarrow{u_{1}}$ and $\overrightarrow{u_{2}}$ are longitudinal i.e. $\overrightarrow{u_{1}} \| \overrightarrow{k_{1}}$ and $\overrightarrow{u_{2}} \| \overrightarrow{k_{2}}$ so that:

$$
\left\{\begin{array}{l}
\overrightarrow{u_{1}}=A_{1} \cos \left(\overrightarrow{k_{1}} \cdot \vec{r}\right)(\vec{\imath}+\vec{\jmath}) \\
\overrightarrow{u_{2}}=A_{2} \cos \left(\overrightarrow{k_{2}} \cdot \vec{r}\right)(\vec{\imath}-\vec{\jmath})
\end{array}\right.
$$

and:

$$
\left\{\begin{array}{l}
u_{x}(x, y)=\left(\overrightarrow{u_{1}}+\overrightarrow{u_{2}}\right) \cdot \vec{\imath}=A_{1} \cos \left(\overrightarrow{k_{1}} \cdot \vec{r}\right)+A_{2} \cos \left(\overrightarrow{k_{2}} \cdot \vec{r}\right) \\
u_{y}(x, y)=\left(\overrightarrow{u_{1}}+\overrightarrow{u_{2}}\right) \cdot \vec{\jmath}=A_{1} \cos \left(\overrightarrow{k_{1}} \cdot \vec{r}\right)-A_{2} \cos \left(\overrightarrow{k_{2}} \cdot \vec{r}\right)
\end{array}\right.
$$

where $A_{1}$ and $A_{2}$ are the amplitudes of the displacements along $(\vec{\imath}+\vec{\jmath})$ and $(\vec{\imath}-\vec{\jmath})$ respectively. The diffracted intensity $I$ is then obtained in reciprocal coordinates $\left(q_{x}, q_{y}\right)$ by taking the squared Fourier Transform of the total electron density $\varrho_{t o t}(x, y)$ which has been beforehand included in a larger volume of zero density to account for the finite size of the sample and get the associated 
squared sinc form factor in the intensity distribution. This new total electron density is called $\varrho_{\text {tot }}^{b}(x, y)$ and $I\left(q_{x}, q_{y}\right)$ thus reads:

$$
I\left(q_{x}, q_{y}\right)=\left|\mathcal{F}\left(\varrho_{t o t}^{b}(x, y)\right)\right|^{2}
$$

The results of this model are shown in Figure 5. We took $N=14,\left\|\overrightarrow{k_{1}}\right\|=\left\|\overrightarrow{k_{2}}\right\|=2 \pi / \lambda$ with $\lambda=5.33 a, A_{1}=A_{2}=0.069 a$. We chose those values in such a way that the resolutions in both real and reciprocal spaces are satisfactory, and used non-trivial fractional quantities to avoid the superposition of eventual artifacts.

The non-modulated and modulated atomic positions are shown in Figure $5 \mathrm{a}$ and $5 \mathrm{~b}$ respectively, and the associated diffractograms in Figure $5 \mathrm{c}$ and $5 \mathrm{~d}$. In the case of a non-modulated structure, the diffraction pattern is very similar to the one found in MAPI in the oKL plane (Figure 1C), with peaks arranged in a square lattice. When the modulation is added, the atomic lattice is buckled, as expected (Figure $5 \mathrm{~b}$ ) and additional peaks appear around Bragg reflections on the diffractogram (Figure 5 d). Superstructure peaks appear around Bragg reflections at reciprocal positions $\pm \overrightarrow{k_{1}}$ or $\pm \overrightarrow{k_{2}}$ or both $\pm \overrightarrow{k_{1}}$ and $\pm \overrightarrow{k_{2}}$, depending on the value of the scalar product between the $\vec{Q}$ vector of the considered Bragg reflection and the displacement direction. When $\vec{Q} \cdot \overrightarrow{u_{p}}=0(p=1$ or 2$)$, the corresponding satellite reflection in the specific direction of $\overrightarrow{k_{p}}$ is not present. Another property of those satellite reflections is that their intensity increases with increasing $\|\vec{Q}\|$ values and at the same time the intensity of the associated Bragg reflections decreases to keep the total intensity of the Bragg and its satellites constant. A zoom of the central region of the diffractogram is shown in Figure 5e. One can see that in this model, the superstructure peaks that appear around the 400 Bragg reflection for instance are very similar to the ones found in the $h=3.98$ plane of MAPI. In addition, the intensity of each of this reflection is not equal, but depends on how far they are located with respect to the reciprocal space origin. Non-equal intensities were indeed observed in the data, but the careful comparison between the measured and calculated intensity distributions requires further studies seen the simplicity of the model taken here compared to the real system. However, in the calculation, the separation distance of the additional peaks from the main Bragg reflection is the same for all Bragg reflections, contrary to what appears in our data. This strong difference actually rules out any explanation based on a modulated structure in this case.

We finally modelled tilted domains of the sample. To do this, a similar 2D square lattice as in the previous simulation was built (Fig 6a) (without modulation: $u_{x}(x, y)=u_{y}(x, y)=0$ ), and two other ones were obtained by applying both a lattice expansion and a rotation by $+3^{\circ}$ and $-3^{\circ}$ (see Fig $6 b, c$ ). The scattering factors were taken different for the lattice at $0^{\circ}$ and for the ones at $\pm 3^{\circ}$ to account for the likely unequal domain sizes of different orientations. The diffracted intensities were computed 
by summing the patterns obtained with the three lattice orientations, taking again the squared Fourier Transform of the total density. The results are shown in Fig $6 \mathrm{~d}$, e. In that case, the additional reflections are also located at lower q values than the Bragg reflections (as the lattice constant is expanded), and the peaks are further apart and from the main Bragg reflection for increasing distance from the center of reciprocal space. Moreover, the additional reflections are always lying in the plane perpendicular to the q-vector of the main Bragg reflection. Those two features are in agreement with our data : the diffraction patterns measured around the 400, 800 and 402 reflections, shown in Fig $4 a, b, c$, can directly be compared to the simulated reflections 200, 400 and 201 of Fig 6 e. This suggests that tilted domains are very likely to explain the measurements in our samples. In the data, the rotation angle $\alpha$ of these sub-domains can be calculated as follows:

$$
\alpha=\tan ^{-1}\left(\frac{\sqrt{\left(\Delta k^{2}+\Delta l^{2}\right)}}{3.98}\right)=0.6^{\circ}
$$

In our data, we measure 4 additional reflections, meaning that to reproduce our data, one should take 4 tilts in 4 directions of space.

However, many other features remain to be understood, especially why is this precise value emerging in the structure, and what is the process leading to it? Are the tilted regions arranged in domains, or do they appear only close to the surface? To answer those questions, additional measurements will be necessary, as well as computations to get additional information about the role of strain for instance. Indeed, the fact that the sample grows in the cubic phase with a (110) orientation of the cubic system and then undergoes the cubic to tetragonal transition, fixes the surface orientation to (100) in the tetragonal system. The surface strain due to this transition could result in both a tilt and an expansion of the lattice in some regions of the sample.

In any case, the exact nature of this additional structure has to be clarified with other experiments and its correlation with the intrinsic electronic properties of MAPI further investigated. If this structural feature appears only near the surface, it could be correlated to different near-surface and bulk electronic and optical features, through a modification of the band structure or double charged surface layers [21].

\section{Conclusion}

We have studied $\left(\mathrm{CH}_{3} \mathrm{NH}_{3} \mathrm{Pbl}_{3}\right)$ single crystals using $\mathrm{x}$-ray diffraction. We have evidenced satellite peaks with a square symmetry in all the different samples studied. This puzzling feature can be addressed with different hypothesis. Here we first considered a periodic lattice displacement model based on a modulated structure with 2 different in-plane modulations of about $20 \mathrm{~nm}$ wavelength in order to describe the measurements. We have found that this model can well describe the 
measurements close the specific 400 Bragg reflection but is not consistent with the measurements performed around the 800 and 402 Bragg peaks. A second scenario involving tilted domains seems to be in better agreement with the different measurements performed around the three Bragg reflections. Another interesting feature is that this additional structure lies in an expanded region of the sample, probably near the sample surface. Further measurements should help understand better these new crystallographic features and eventually relate them to other puzzling features of this system like for instance a relatively good tolerance to defects or the long carrier diffusion lengths and efficient charge collection observed in this material.

\section{Acknowledgments}

O. Plantevin acknowledges Angelo Malachias (Universidade Federal de Minas Gerais, Belo Horizonte, Brazil) for the discussion about data interpretation and the "tilt" model.

This work was supported by the LabEx PALM (ANR-10-LABX-0039-PALM), and IRS MOMENTOM (Université Paris-Saclay).

This work has received funding from the European Union's Horizon 2020 programme, through a FET Open re- search and innovation action under grant agreement No. 687008. The information and views set out in this article are those of the authors and do not necessarily reflect the official opinion of the European Union. Neither the European Union institutions and bodies nor any person acting on their behalf may be held responsible for the use which may be made of the information contained herein.

\footnotetext{
${ }^{1}$ Y. Kawamura, H. Mashiyama, K. Hasebe, J. Phys. Soc. Jap. 71 (7) (2002) 1694.

${ }^{2}$ P.S. Whitfield, N. Herron, W.E. Guise, K. Page, Y. Q. Cheng, I. Mila, M.K. Crawford, Sci. Rep. 6 (2016) 35685 .
}

${ }^{3}$ M.T. Weller, O.J. Weber, P.F. Henry, A.M. Di Pumpo, T.C. Hansen, Chem. Commun. 2015, 51, 41804183 .

${ }^{4}$ M.I. Saidaminov, A.L. Abdelhady, B. Murali, E. Alarousu, V.M. Burlakov, W. Peng, I. Dursun, L. Wang, Y. He, G. Maculan, A. Goriely, T. Wu, O.F. Mohammed, and O.M. Bakr, Nature Comm. 6 (2015) 7586.

${ }^{5}$ W.J. Yin, T. Shi, Y. Yan, Appl. Phys. Lett. 104 (2014) 063903.

${ }^{6}$ W.-J. Yin, J.-H. Yang, J. Kang, Y. Yan, S.-H. Wei, J. Mater. Chem. A 3 (2015) 8926.

${ }^{7}$ Y. Dang, Y. Liu, Y. Sun, D. Yuan, X. Liu, W. Lu, G. Liu, H. Xia, X. Tao, CrystEngComm (2015), 17, 665.

${ }^{8}$ X. Chi, K. Leng, B. Wu, D. Shi, Y. Choy, Z. Chen, Z. Chen, X. Yu, P. Yang, O.-H. Xu, T. C. Sum, A. Rusydi, and K.P. Loh, Adv. Optical Mater. (2018) 1800470. 
${ }^{9}$ B. Murali, S. Dey, A.L. Abdelhady, W. Peng, E. Alarousu, A.R. Kirmani, N. Cho, S.P. Sarmah, M.R. Parida, M.I. Saidaminov, A.A. Zhumekenov, J. Sun, M.S. Alias, E. Yengel, B.S. Ooi, A. Amassain, O.M. Bakr, O.F. Mohammed, ACS Energy Lett., (2016), 11119.

${ }^{10}$ L. She, M. Liu, D. Zhong, ACS Nano (2015) 506420.

${ }^{11}$ H. Diab, C. Arnold, F. Lédée, G. Trippe-Allard, G. Delport, C. Vilar, F. Bretenaker, J. Barjon, J.-S. Lauret, E. Deleporte and D. Garrot, J. Phys. Chem. Lett. 8 (2017) 2977.

${ }^{12}$ V. L. R. Jacques, S. Ravy, D. Le Bolloc'h, E. Pinsolle, M. Sauvage-Simkin and F. Livet, Phys. Rev. Lett. 106 (2011) 065502.

${ }^{13}$ V. L. R. Jacques, D. Carbone, R. Ghisleni and L. Thilly, Phys. Rev. Lett. 111 (2013) 065503.

${ }^{14}$ V. L.R. Jacques, D. Le Bolloc'h, S. Ravy, C. Giles, F. Livet and S. B. Wilkins, Eur. Phys. J. B 70 (2009) 317.

${ }^{15}$ G.Grüner, Density Waves in Solids, Addison Wesley (1994)

${ }^{16}$ XX16 S. van Smaalen, Incommensurate Crystallography, Oxford University Press (2007).

${ }^{17}$ D. Le Bolloc'h, V. L. R. Jacques, N. Kirova, J. Dumas, S. Ravy, J. Marcus and F. Livet, Phys. Rev. Lett. 100 (2008) 096403.

${ }^{18}$ V. L. R. Jacques, D. Le Bolloc'h, S. Ravy, J. Dumas, C. V. Colin and C. Mazzoli, Phys. Rev. B 85 (2012) 035113.

${ }^{19}$ Y. Guo, O. Yaffe, D.W. Paley, A.N. Beecher, T.D. Hull, G. Szpak, J.S. Owen, L.E. Brus, and M.A. Pimenta, Phys. Rev. Mat. 1 (2017) 042041(R).

${ }^{20}$ I.P. Swainson, Acta Cryst. B61 (2005),616.

${ }^{21}$ S.P. Sarmah, V. Burlakov, E. Yengel, B. Murali, E. Alarousu, A.M. El-Zohry, C. Yang, M. S. Alias, A.A. Zhumekenov, M.I. Saidaminov, N. Cho, N. Wehbe, S. Mitra, I.A. Ajia, S. dey, A.E. Esam Mansour, M. Abdelsamie, A. Amassian, I.S. Roqan, B.S. Ooi, A. Goriely, O.M. Bakr, O.F.

Mohammed, Nano Lett. (2017) 172021. 


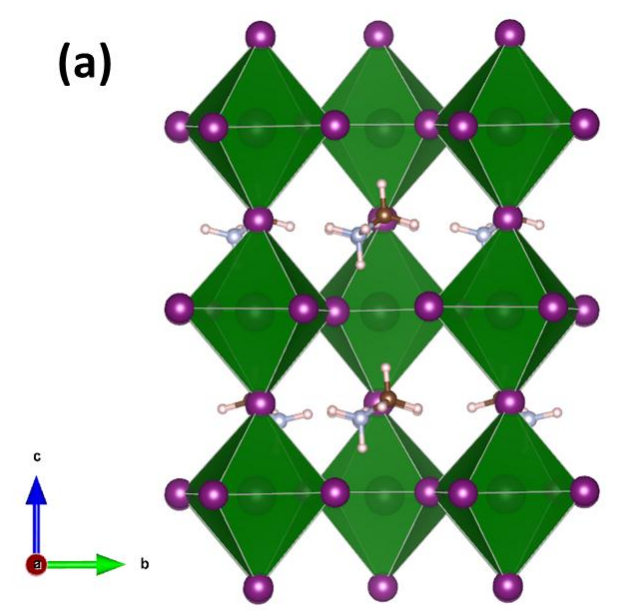

(c)

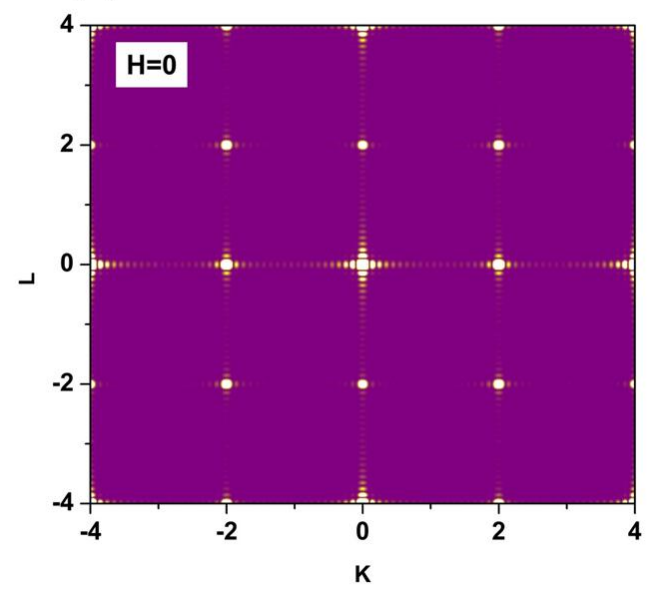

(b)

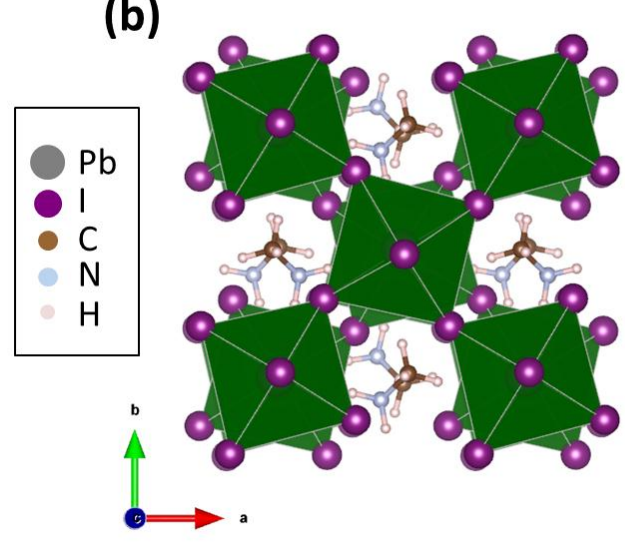

(d)

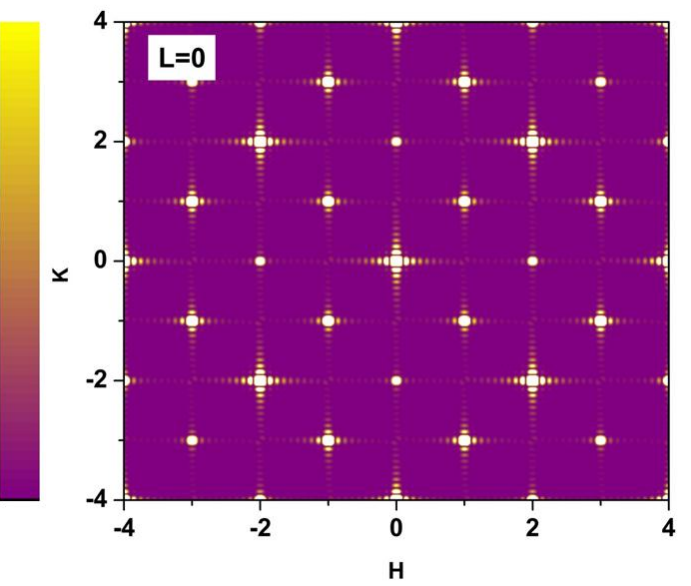

Figure 1 : Structure of MAPI in the tetragonal phase, $(a)$ in the $(b, c)$ plane and in $(b)$ in the $(a, b)$ plane.

Corresponding calculated diffraction patterns calculated, (c) in the oKL reciprocal plane, and (d) in the HKo reciprocal plane. The finite number of cells taken in the calculation gives rise to the fringes around Bragg reflections (squared sinc function form factor related to a cubic volume).

(a)

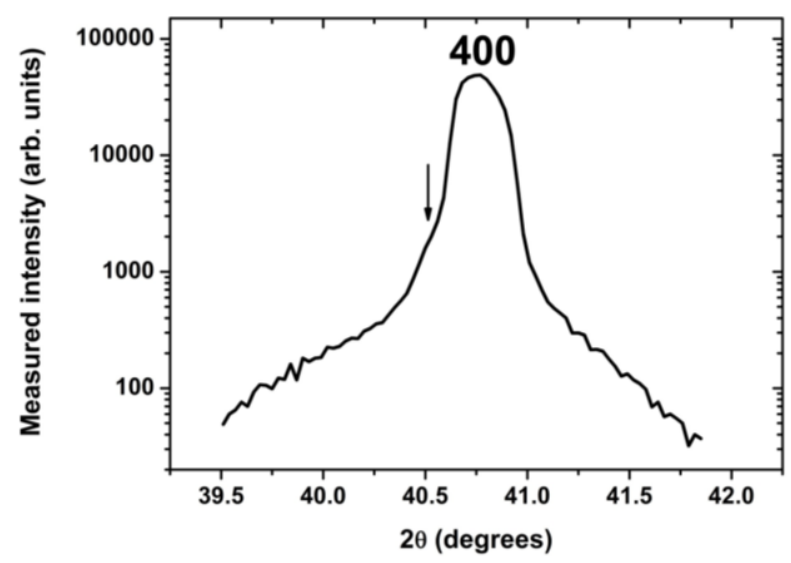

(b)

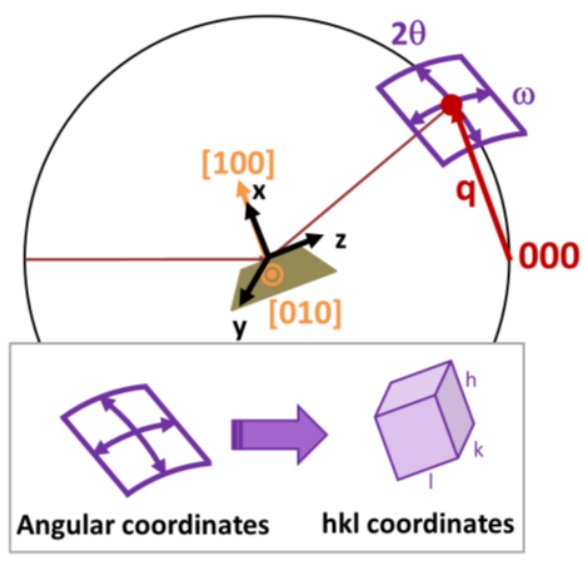

Figure 2 : (a) Longitudinal $\theta-2 \theta$ scan performed around the 400 specular Bragg reflection, with a $8 \mathrm{keV}$ beam generated by a rotating anode (log scale). A shoulder is visible on the lower angles from the 400 position, but not at higher angles. (b) Scheme of the experiment and ${ }_{3} D$ data recording in the vertical scattering plane. $2 D$ angular maps were recorded at different $\omega$ and $\chi$ angles by rotating the sample around the $y$-axis and $z$-axis respectively. 
The out-of-scattering-plane plane volume is reached using $\chi$ (not shown on the figure). Such $2 D$ angular maps were recorded at several detector positions $2 \theta$, thus making a $3 D$ volume, that was then converted into a new $3 D$ volume indexed in hkl coordinates associated to the sample Fourier space.

(a)

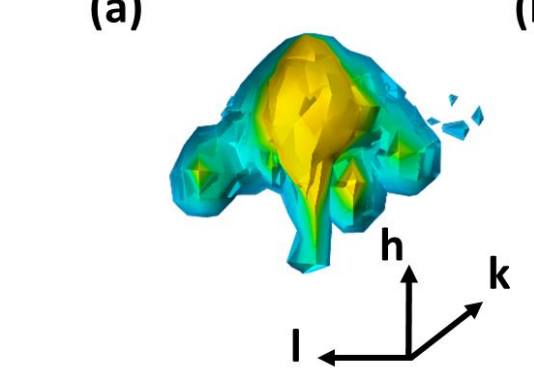

(c)

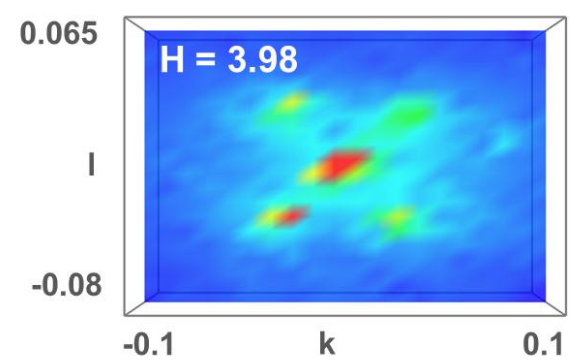

(b)

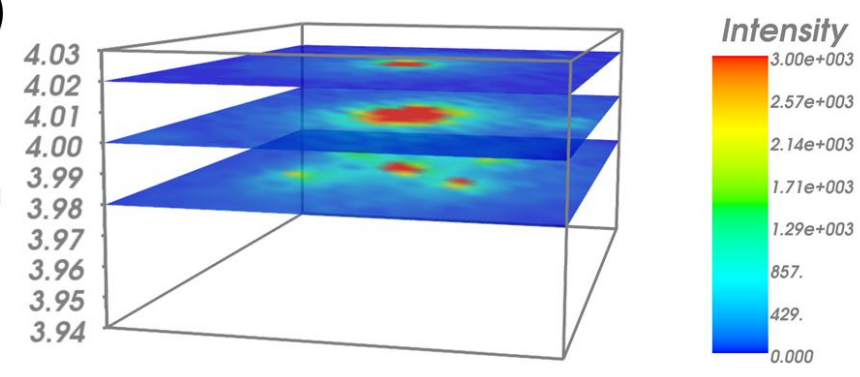

(d)

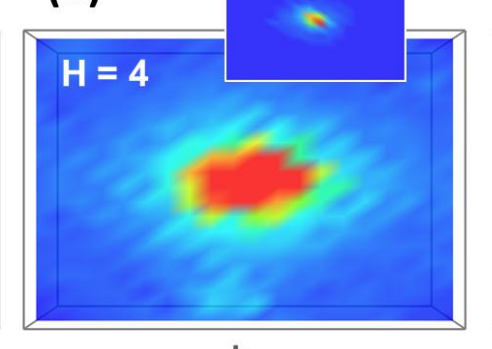

k (e)

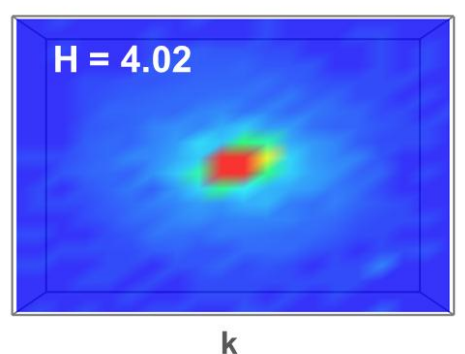

Figure 3 : (a) Intensity recorded in the 3D volume around the 400 Bragg reflection in the hkl coordinates system, with $8.04 \mathrm{keV} x$-rays. Several iso-surfaces are represented, and reveal both the main 400 Bragg reflection (upper strong intensity region), and the 4 satellites in a lower- $h$ plane. (b) Same hkl volume with intensity maps shown at three specific $h$ values, at and around the 400 reflection: $h=3.98, h=4$ and $h=4.2$. The intensity scale has been limited to $3.10^{3}$, ie $1 / 100$ of the maximum Bragg intensity, to better highlight the 4 extra peaks in the $h=3.98$ plane. (c)-(e) Same $k l$ maps recorded at $h=3.98, h=4$ and $h=4.2$, with the same scale as in (b). The extra peaks appear in (c) at $\pm(\Delta k \pm \Delta l)$, with $\Delta k \approx \Delta l=0.030$. Inset of $(d)$ : same map scaled with the maximum intensity. Note that the small elongations are due to interpolation after conversion of the angular volume into hkl volume.
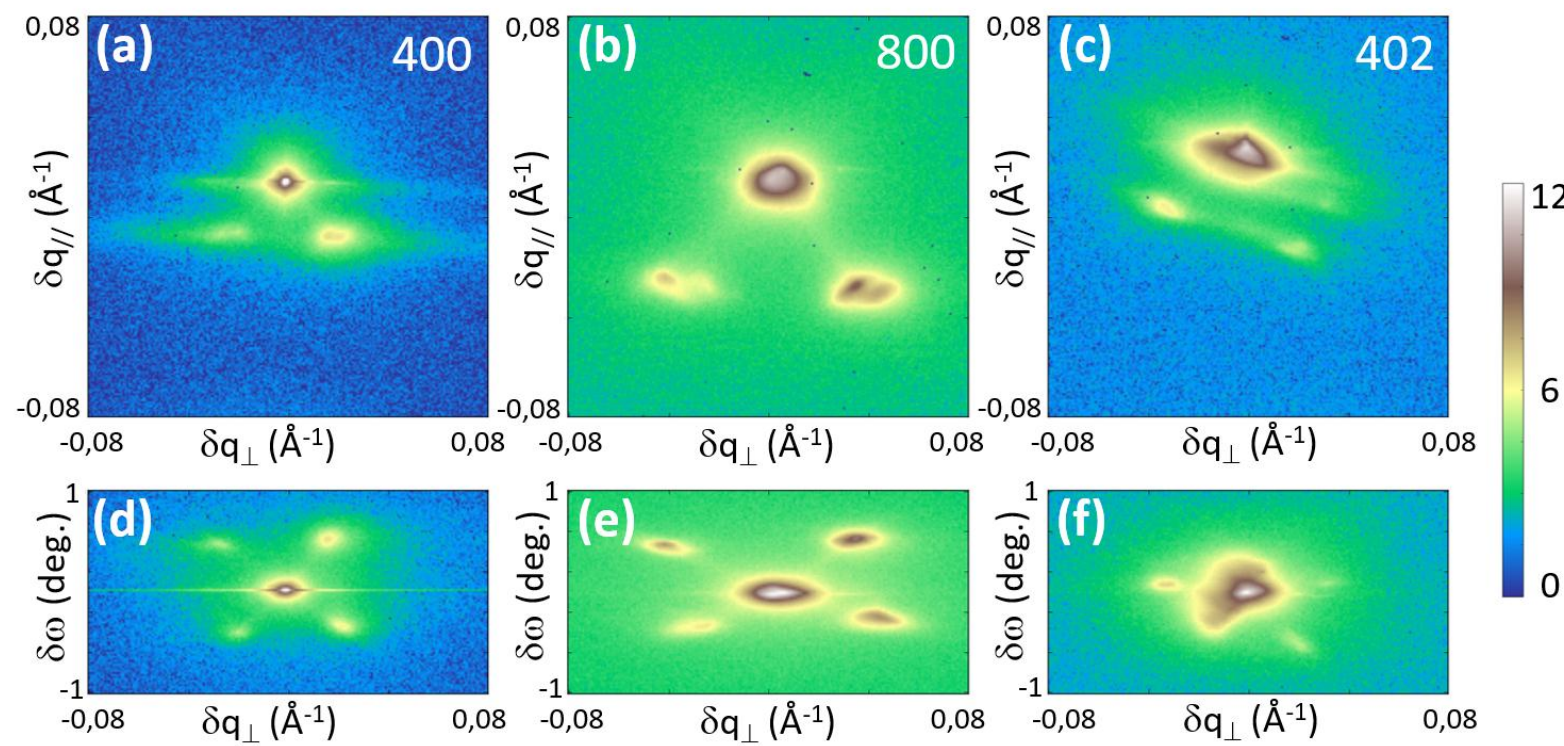

Figure 4: The diffracted intensity was obtained in $3 D$ around the 400, 800 and 402 Bragg reflections by rocking the sample around $\omega$ and recording intensity on a $2 D$ detector, measured with $18.48 \mathrm{keV} x$-rays. The vertical and 
horizontal directions of the detector correspond to directions in reciprocal space called $q_{/ /}$and $q_{\perp}$ respectively. These $3 D$ angular volumes were then projected along the rocking direction $\omega$ (upper line), and along $q_{/ /}$(lower line) around the three Bragg reflections: (a) and (d) around the 400 reflection; (b) and (e) around the 800 reflection; (c) and (f) around the 402 reflection. The intensity scale is logarithmic and identical for all maps.

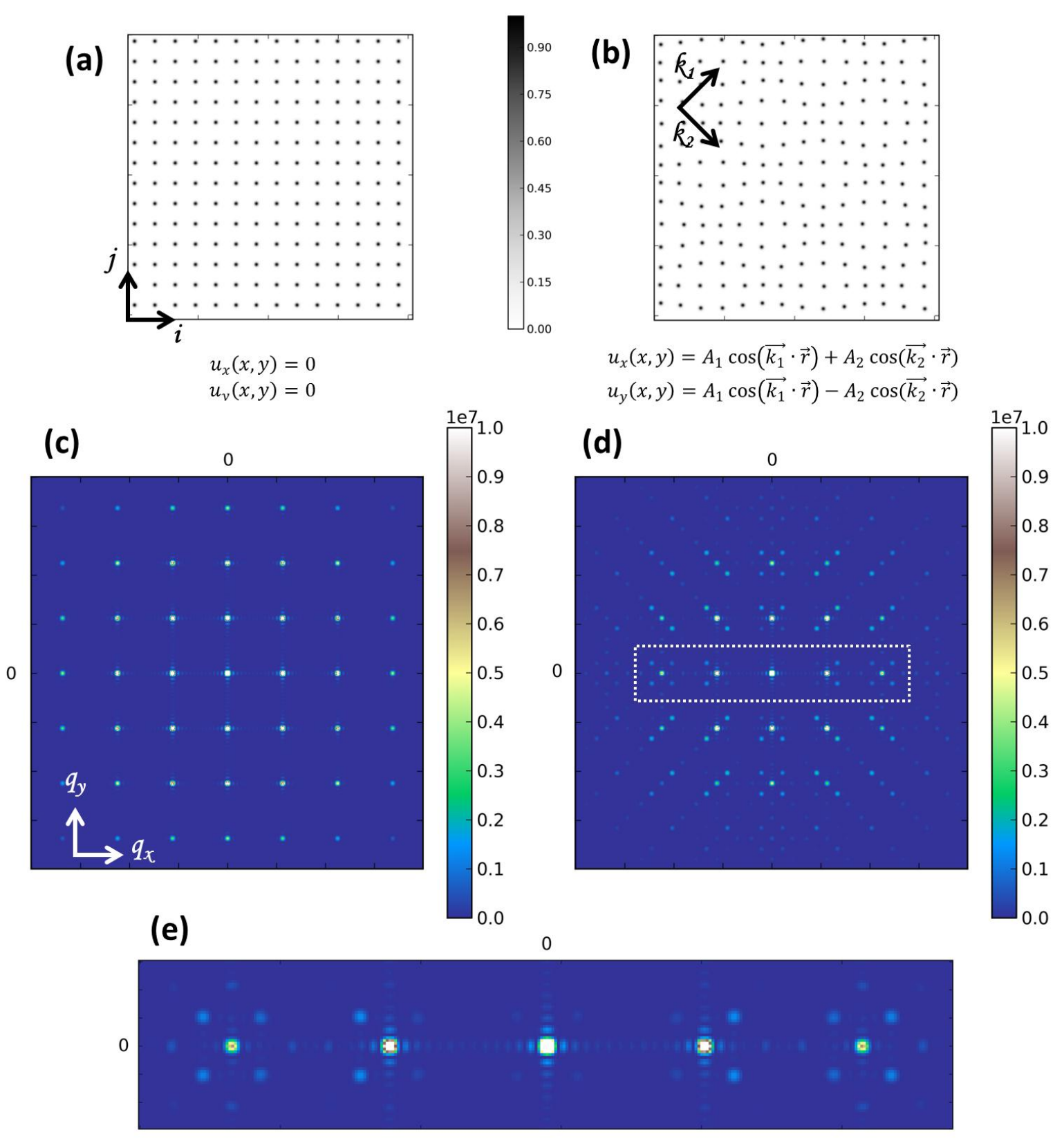

Figure 5 : Simulations of a $2 D$ single-atomic square crystal and associated diffraction patterns. (a) Perfect square lattice obtained by the convolution of a Dirac comb with an on-site Gaussian-like electron density. (b) Same lattice obtained using periodic displacements along the two diagonal directions (see text). (c) and (d) Diffractograms associated to the lattices shown in (a) and (b) respectively, obtained by calculating the squared Fourier Transform of the real space total electron density (see text). The linear intensity scale has been truncated to the third of the maximum intensity. The form factor appears on each Bragg reflection as a squared sinc modulation. For the double-modulated crystal, additional peaks appear around Bragg reflections along $k_{1}$ and $k_{2}$, with selection rules depending on the displacement direction. (e) Zoom on the central region of (d). First and second order peaks are visible, and the intensity of the satellites is higher at higher $q$ wavevectors. 
(a)

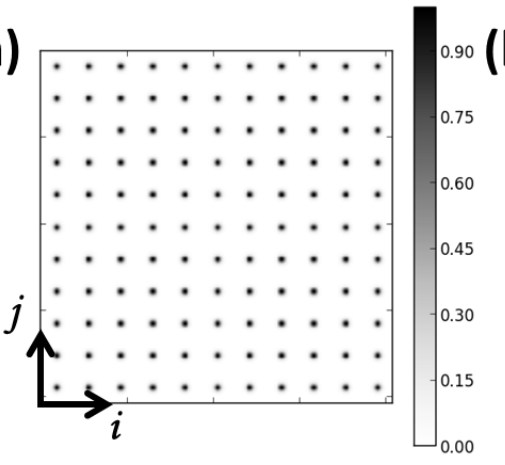

(d)

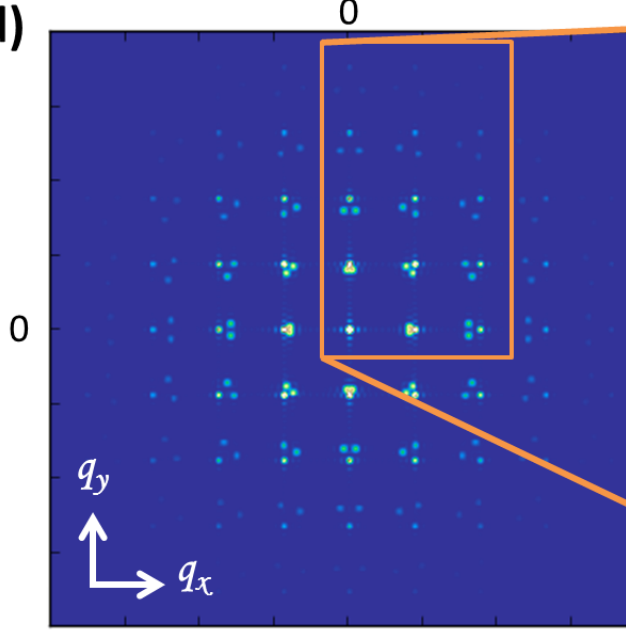

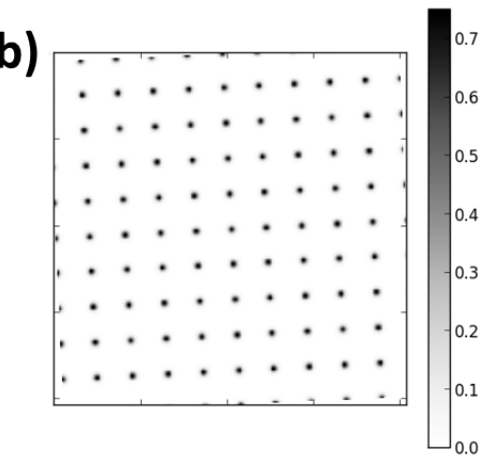

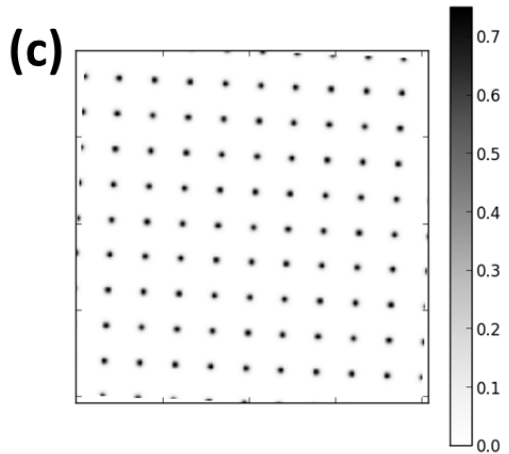

400

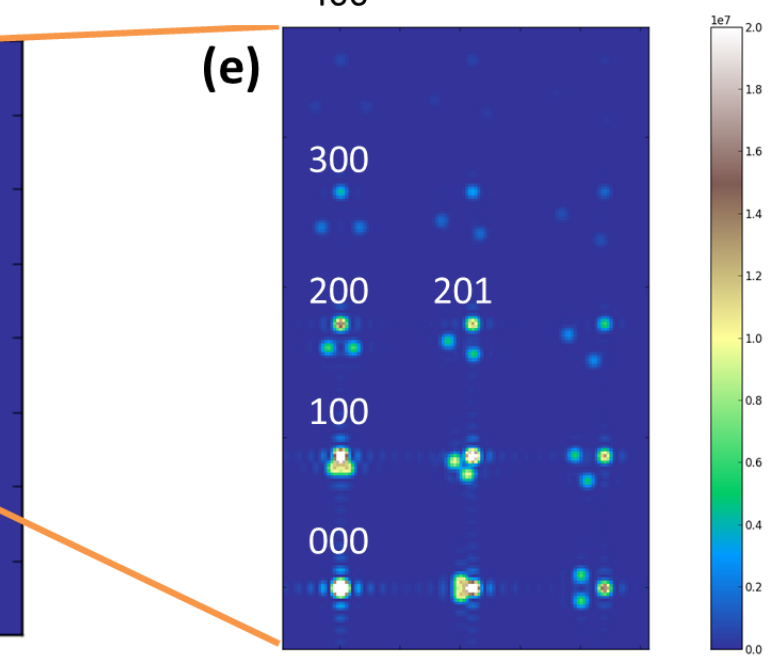

Figure 6: Simulation of $2 D$ square lattices obtained by the convolution of a $2 D$ Dirac comb with Gaussian-like electron densities: (a) without tilt ; (b) with $a+3^{\circ}$ tilt; (c) with $a-3^{\circ}$ tilt. The on-site electron density is different for the untilted and tilted lattices to account for the eventual non-equal volumes in which they respectively appear. (d) Computed reciprocal space map corresponding to the superposition of the three lattices of different orientation, obtained by summing the Fourier transforms of each real-space lattice. (e) Zoom on a region of the reciprocal space map showing that the expected peaks are much further apart for increasing $q$-values, and that their orientation depends on the direction of reciprocal space considered. 